\title{
Heparin-based coacervate of bFGF facilitates peripheral nerve regeneration by inhibiting endoplasmic reticulum stress following sciatic nerve injury
}

\author{
Rui $\mathrm{Li}^{1, *}$, Shuang Zou ${ }^{1, *}$, Yanqing Wu ${ }^{2}$, Yiyang $\mathrm{Li}^{1}$, Sinan Khor ${ }^{5}$, Yuqin Mao ${ }^{1}$, \\ Huacheng $\mathrm{He}^{2}$, Ke Xu ${ }^{2}$, Hongyu Zhang ${ }^{1}$, Xiaokun $\mathrm{Li}^{2}$, Jian Wang ${ }^{3}$, Huai Jiang ${ }^{4}$, Qike \\ $J^{3}{ }^{4}$, Qingsong $\mathbf{Y e}^{1}$, Zhouguang Wang ${ }^{1}$ and Jian $\mathrm{Xiao}^{1}$ \\ ${ }^{1}$ Molecular Pharmacology Research Center, School of Pharmaceutical Sciences, Wenzhou Medical University, Wenzhou, \\ Zhejiang 325035, China \\ ${ }^{2}$ The Institute of Life Sciences, Wenzhou University, Wenzhou, Zhejiang 325035, China \\ ${ }^{3}$ Department of Peripheral Neurosurgery, The First Affiliated Hospital of Wenzhou Medical University, Wenzhou, Zhejiang \\ 325035, China \\ ${ }^{4}$ Department of Neonatology, The Second Affiliated Hospital of Wenzhou Medical University, Wenzhou, Zhejiang 325035, China \\ ${ }^{5}$ Department of Molecular Pharmacology, Albert Einstein College of Medicine, Bronx, NY 10461, USA \\ *These authors have contributed equally to this work \\ Correspondence to: Zhouguang Wang, email: huaikongwang@126.com \\ Jian Xiao, email: xfxj2000@126.com \\ Keywords: peripheral nerve injury, controlled release, basic fibroblast growth factor, endoplasmic reticulum stress, coacervate \\ Received: March 07, $2017 \quad$ Accepted: April 29, $2017 \quad$ Published: May 29, 2017
}

Copyright: Li et al. This is an open-access article distributed under the terms of the Creative Commons Attribution License 3.0 (CC BY 3.0), which permits unrestricted use, distribution, and reproduction in any medium, provided the original author and source are credited.

\section{ABSTRACT}

Creating a microenvironment at the injury site that favors axonal regrowth and remyelinationis pivotal to the success of therapeutic reinnervation. The mature myelin sheath of the peripheral nervous system depends on active participation of Schwann cells to form new cytoskeletal components and tremendous amounts of relevant neurotrophic factors. In this study, we utilized a new biomaterial for growth factor delivery consisting of a biocompatible polycation, poly(ethylene argininylaspartatediglyceride) and heparin. It is capable of binding a variety of growth factors to deliver basic fibroblast growth factor (bFGF) through polyvalent ionic interactions for nerve repair. In vitro assays demonstrated that the bFGF loading efficiency reached $\mathbf{1 0} \mathrm{\mu g}$ and this delivery vehicle could control the release of bFGF. In vivo, the coacervate enhanced bFGF bioavailability, which improved both motor and sensory function. It could also acceleratemyelinated fiber regeneration and remyelination and promote Schwann cells proliferation. Furthermore, the neuroprotective effect of bFGF-coacervate in sciatic nerve injury was associated with the alleviation of endoplasmic reticulum stress signal. This heparin-based delivery platform leads to increased bFGF loading efficiency and better controls its release, which will provide an effective strategy for peripheral nerve injury regeneration therapy.

\section{INTRODUCTION}

Traffic accidents, trauma, and tumor resection can result in peripheral nerve injury (PNI). PNI is a common and serious health problem that can result in restricted activity and long-term disability. It was previously reported that about $3 \%$ of trauma patients are affected by PNI and require surgery [1]. At present, the gold standard treatment for peripheral nerve repair is autologous nerve grafting, but this technique is limited by tissue availability 
as well as immunological rejection [2]. Therefore, it is of great therapeutic interest to develop an alternative to the conventional grafting therapeutic method that provides a protected microenvironment that can spontaneously promote axonal sprouting and regeneration as well as supplements adequate growth factors (GFs) at the site of the lesion.

Neurotrophic factors (NTFs), including basic fibroblast growth factor (bFGF), have been widely used as a therapeutic strategy for preventing the PNI progression $[3,4]$. bFGF is composed of 146 amino acids and belongs to a 22-member family of polypeptides. Recent advances inbiological materials have given way to a wealth of research about bFGF facilitating axon growth and nerve regeneration after PNI $[5,6]$. However, bFGF has a very short half-life, and it is vulnerable to a variety of proteolytic cleavage events that would inactivate it in bodily fluids [7, 8]. Based on insufficient endogenous bFGF and its high affinity for heparin [9], we prepared a protein delivery coacervate to bind bFGF via charge interaction consisting of polycation-Poly(ethylene argininylaspartatediglyceride) (PEAD) and heparin [10]. This coacervate not only controls bFGF release but also maintains the bFGF's endogenous bioactivity [11]. Thus, this ternary complex could prove to be advantageous in treatments of complicated and refractory diseases including PNI.

Since the mechanisms of nerve regeneration are complex and not well understood, we wanted to explore how bFGF-Coacervate affects sciatic nerve injury. Endoplasmic reticulum stress (ERS) has been implicated in the pathogenesis of neurodegenerative disorders such as diabetic peripheral neuropathy (DPN), spinal cord injury (SCI), and cerebral ischemia-reperfusion (I/R) injury [1214]. The endoplasmic reticulum (ER) is a membranous network in eukaryotic cells that functions in protein packaging and calcium storage [14]. Some of the events that lead to an imbalance between protein synthesis and protein folding can disrupt cellular homeostasis, leading to ERS [15]. To counteract ERS, cells activate the unfolded protein response (UPR) through a signal transduction cascade to restore ER homeostasis [16]. Because of the dearth of literature concerning the relationship between ERS and PNI, we sought to investigate this relationship and bFGF-Coacervate regulation.

Using the sciatic nerve crush injury model in rats, this work examined the beneficial effect of [PEAD:heparin] vehicle combined with bFGF on functional recovery and nerve regeneration. We also illustrated how the therapeutic effects of bFGF-Coacervate are mediated by the ERS-mediated axonal growth/atrophy signaling pathway. The detailed technology roadmap of this experimental design is shown in Figure 1. Overall, this study may have bearing on therapeutic strategies for promoting neural repair following PNI in clinical populations.

\section{RESULTS}

\section{Characterization of bFGF-Coacervate}

As a new delivery vehicle, [PEAD:heparin] interacts strongly with bFGF to form bFGF-Coacervate (or called [PEAD:heparin: bFGF] coacervate) through charge interactions [17]. This was visualized when the clarified heparin-bFGF solution became turbid upon addition of PEAD. However, after $24 \mathrm{~h}$ of standing, bFGFCoacervateprecipitated down to the bottom (Figure 2A). It is known that the maximum binding mass ratio of PEAD/ heparin is 5 by DMB assay [11], but the loading efficiency of [PEAD:heparin] binding to bFGF is unknown. To clarify this, $1 \mu \mathrm{g}, 5 \mu \mathrm{g}$ and $10 \mu \mathrm{g}$ of bFGF were loaded into the [PEAD:heparin] matrix. Western blot results indicated that the amount of bFGF in the coacervate was almost the same as in the loading solution (Figure 2B) and thus, our choice of vehicle had a greater bFGF loading efficiency. We further collected the supernatantat indicated time points to determine bFGF concentration and draw the cumulative release profile of bFGF-Coacervate over 35 days (Figure 2C). There was a near linear, sustained release of bFGF for 28 days, after which it decreased and plateaued in the following days. At the end of the 35 th day, approximately $60 \%$ of residual bFGF was unreleased. Overall, the release curve of bFGF-Coacervate indicated that this matrix can efficiently control the release of incorporated $\mathrm{bFGF}$.

\section{bFGF-Coacervate promotes motor and sensory recovery after $\mathrm{PNI}$}

We evaluated whether bFGF-Coacervate could promote motor and sensory recovery through out the 4-week period using the walking track analysis and hot plate test. The SFI value was not significantly different among the four crushed groups before week 2, although there was a trend toward a time-dependent increase. From week 3, the SFI value in each of the four crushed groups was significantly different. Locomotor performance in the bFGF animals was superior to the PNI and vehicle animals but inferior to the bFGF-Coacervate animals. Moreover, the superior recovery in the bFGF-Coacervate group continued through week 4 (Figure $3 \mathrm{~B}, \mathrm{p}<0.01$ ). Paw withdrawal latency as observed via the hot plate test showed nearly the same trend as the walking track analysis (Figure 3C). This demonstrates that bFGF-Coacervate can persistently improve the recovery of both motor and sensory function after crush injury, and the recovery effect is greater than routinely administrated bFGF. 


\section{bFGF-Coacervate enhances myelinated fiber} regeneration and remyelination at $\mathbf{3 0}$ days postinjury

To assess whether bFGF-Coacervate is capable of enhancing nerve fiber regeneration and remyelination, histomorphometrical analysis was performed at the distal portion of the injured site after 30 days restoration. H\&E and TB sections showed that the regenerated nerve fibers in the bFGF-Coacervate and bFGF groups were denser, more compact, and uniform in comparison to the nerve fibers in the PNI and vehicle groups, which were small and irregular (Figure 4A and 4B). Importantly, we observed thin thickness and scattered density of regenerated myelin sheaths in the PNI and vehicle groups by TEM. Thicker, denser myelin sheaths were noted in the bFGF group, but myelin sheaths in the bFGF-Coacervate administration animals were the most thick and dense (Figure 4C). In addition, statistical analysis revealed that the mean axonal diameters and myelin thickness in with bFGF-Coacervate were the greatest (Figure 4D and 4E), although there was significantly statistical difference between bFGFgroup and vehicle groups as well. Taken together, these results show that bFGF-Coacervate had a beneicial effect on myelinated fiber regeneration and remyelination.

\section{bFGF-Coacervate accelerates axonal growth and promotes Schwann cell (SC) proliferation}

Following injury to a peripheral nerve, SCs undergo dedifferentiation and proliferation with the characteristic expression of glial fibrillaryacid protein (GFAP) [18]. NF200 is the heavy subunit of neurofilaments for both larger and smaller axons. To further determine whether bFGFCoacervate promotes SC proliferation and accelerates new axon growth, the expression of GFAP and NF-200 were examined by immunohistochemistry in injured sciatic nerve transections. As shown in Figure $4 \mathrm{~A}$ and $4 \mathrm{~B}$, there was decreased GFAP and NF-200 signal in the PNI and vehicle groups. bFGF treatment could reverse these changes somewhat, but this increase was inferior to that with bFGFCoacervate injection. Quantitative analysis also showed

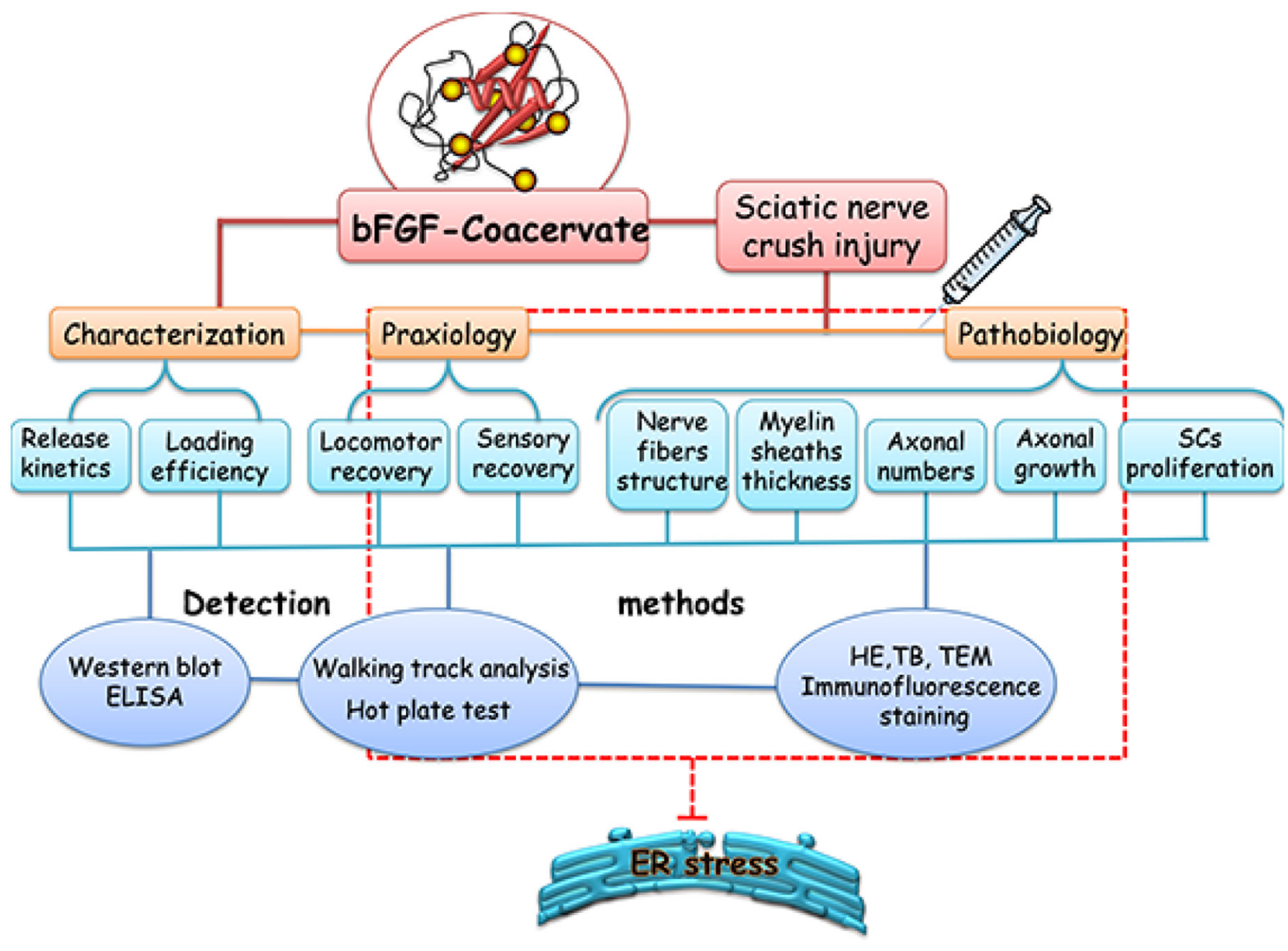

Figure 1: A schematic diagram of the experimental design. We firstly prepared the [PEAD:heparin: bFGF] and detect its property through Western blot and ELISA methods. Next, this bFGF-coacervate was applied to repair the crush injury model of wistar rats via only one injection in the lesion region and analysis the functional improvement and nerve regeneration after 30 days recovery. Lastly, we demonstrated this excellent therapeutic effect was related to the inhibition of prolonged ERS in injuried tissue by Western blot. 
A

C
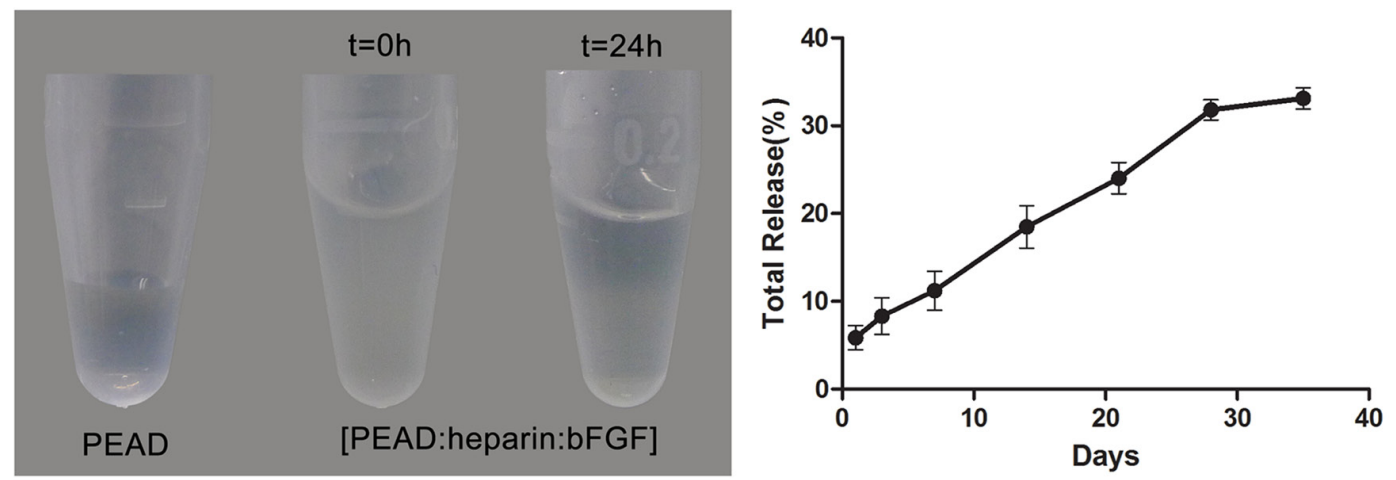

B

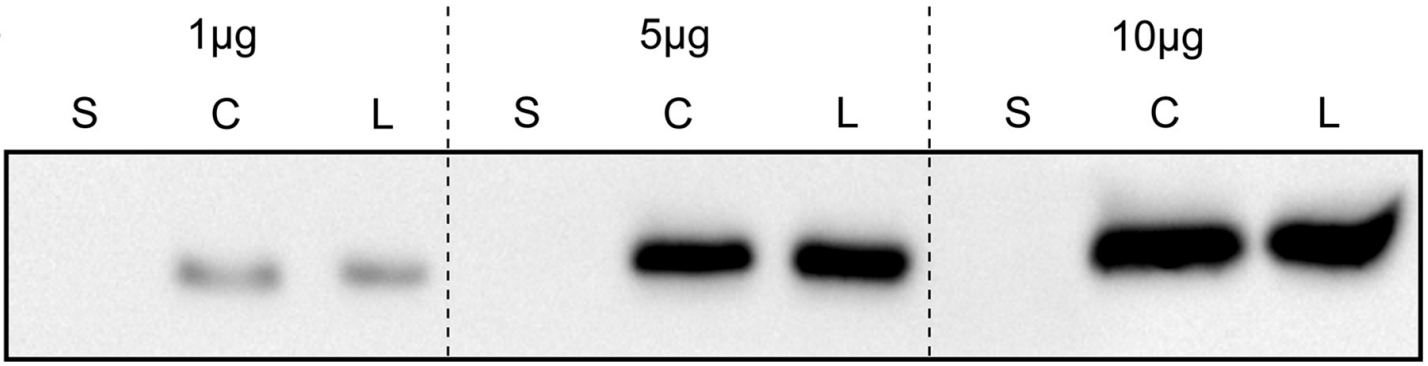

Figure 2: Properties of the [PEAD:heparin] vehicle binding to bFGF. (A) In the preparation of bFGF-coacervate, the precipitation particles settle down to the bottom after $24 \mathrm{~h}$ standing. (B) Western blot analysis of the loading efficiency of bFGF into the coacervate $(500 \mu \mathrm{g}$ PEAD, $100 \mu \mathrm{g}$ heparin, bFGF range tested: $1-10 \mu \mathrm{g})$. S: bFGF in the supernatant after centrifugation. C: bFGF in the settled coacervates. L: total amount of bFGF in the loading solution. (C) bFGF was released from the coacervate into saline at $25^{\circ} \mathrm{C}$. The percentage of release over 35 days was quantified by ELISA. The data represent the means $\pm \mathrm{SD}$ ( $\mathrm{n}=3$ per group).

A
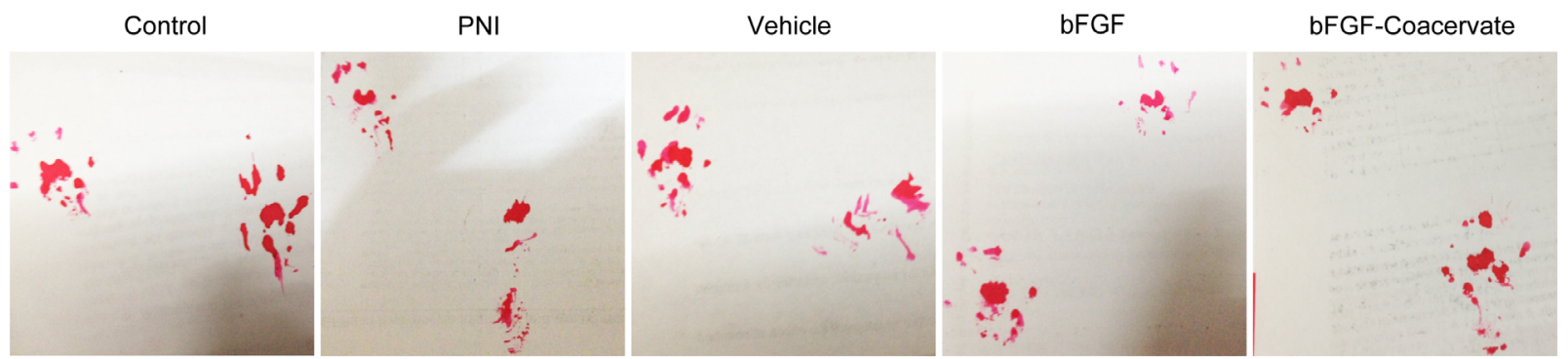

B

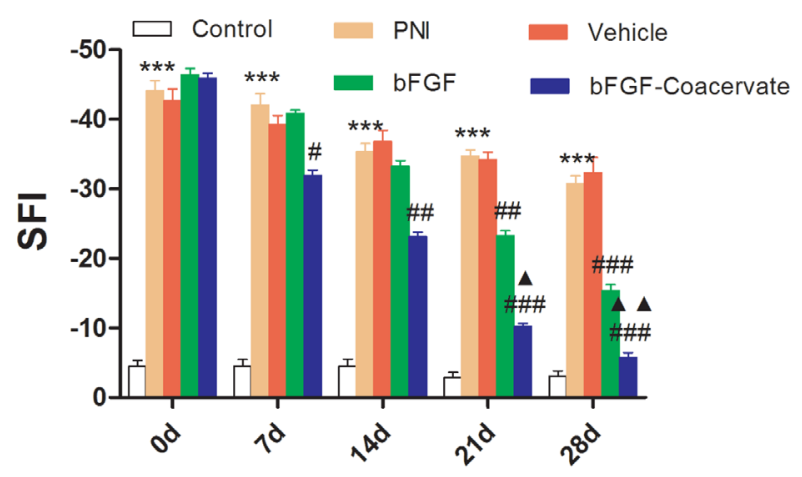

Time (days)

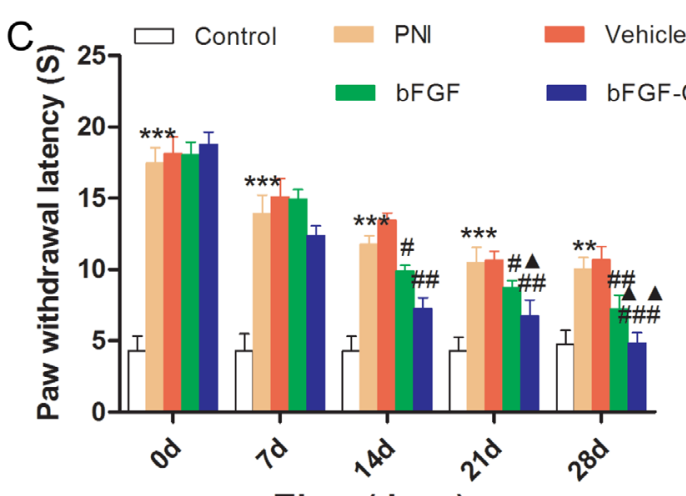

Time (days)

Figure 3: Locomotor and sensory recovery in each group were evaluated by walking track analysis and hot plate test. (A) Representative pictures of rat footprint at 28 days of recovery after sciatic nerve crush. (B) Motor recovery was evaluated using the SFI analysis at $0,7,14,21$ and 28 days after surgery. (C) Statistical analysis of paw withdrawal latency before (0 day) and at indicated time points. ${ }^{* * *} \mathrm{P}<$ 0.001 compared with control group, ${ }^{\#} \mathrm{P}<0.05,{ }^{\#} \mathrm{P}<0.01,{ }^{\# \#} \mathrm{P}<0.001$ versus $\mathrm{PNI}$ group, ${ }^{\mathbf{\Lambda}} \mathrm{P}<0.05,{ }^{\boldsymbol{\Delta}} \mathrm{P}<0.01$ versus bFGF group. 
that tissue from the bFGF-Coacervate treated samples had significantly higher GFAP and NF-200 expression than the bFGF group (Figure $5 \mathrm{C}$ and $5 \mathrm{D}, p<0.05$ ). These results suggesting that bFGF-Coacervate increases the regenerative capacity of injured peripheral nerves.

\section{The regenerative effect of bFGF-Coacervate is mediated by inhibiting ERS after acute PNI}

It has been reported that excessive or prolonged activation of ERS can cause neuronal disorders, eventually leading to cellular apoptosis [16, 19]. However, it remains unclear whether ERS prevents the functional and structural recovery of an injured sciatic nerve and whether bFGF-Coacervate treatment can regulate ERS associated with PNI. These hypothesis in our study were detected by Western blot. As demonstrated in Figure 6, the levels of ERS-associated proteins GRP-78, ATF-6, XBP-1, Cleaved-caspase 12 and CHOP were increased significantly after $30 \mathrm{~d}$ contusion. On the contrary, free bFGF combined with/without [PEAD:heparin] vehicle treatment could reduce the expression of these ERS markers. The bFGF-Coacervate samples displayed the greatest reduction in ERS markers. These changes were corroborated by statistical analysis of the band density (Figure 6B, 6C, 6D, 6E and 6F), which indicated that

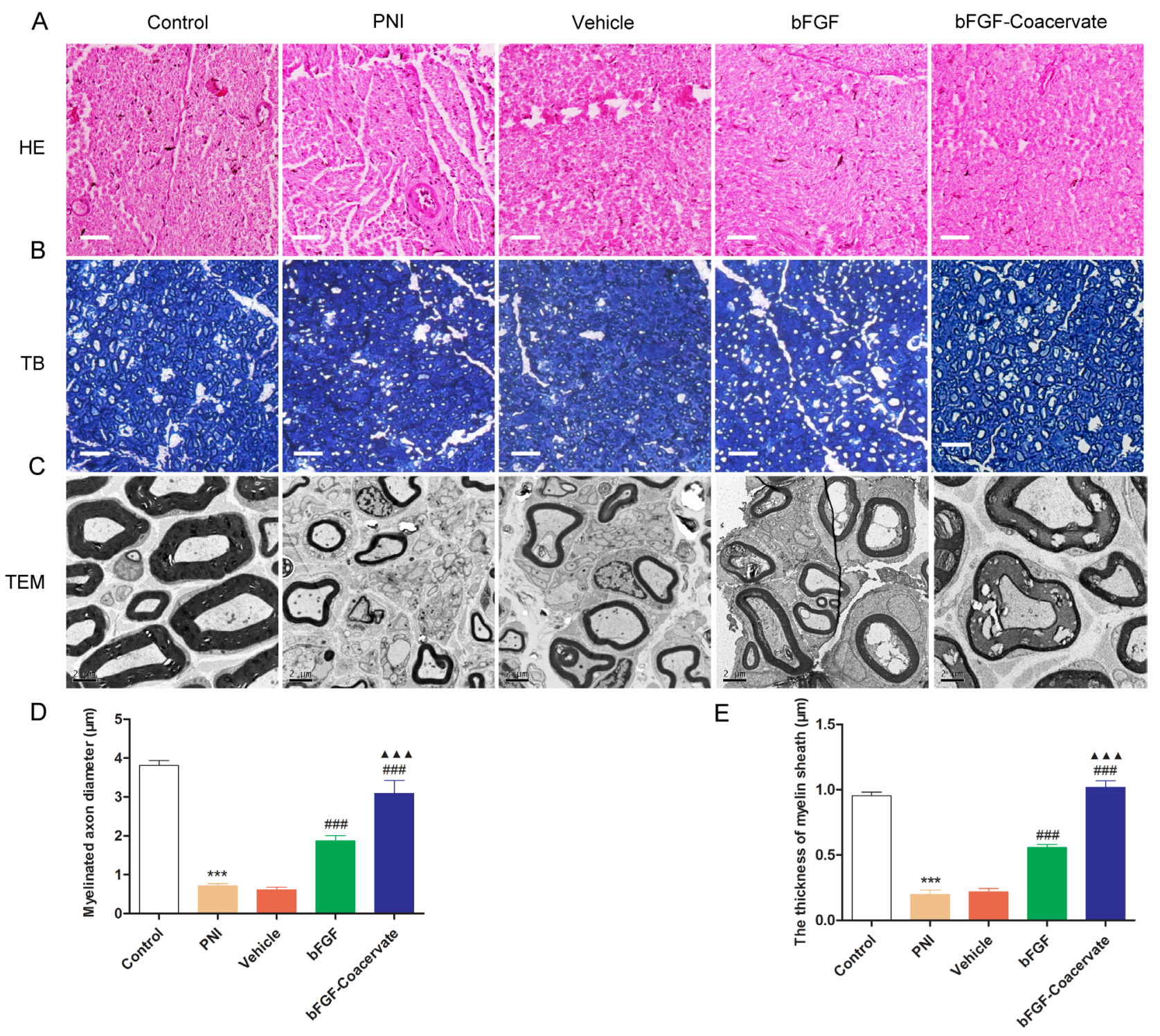

Figure 4: Histological investigation and morphometric analysis at 30 days after crush injury. (A) H\&E staining of sciatic nerve. Light micrographs of trans-sections in the five groups. Scale bar $=50 \mu \mathrm{m}$. (B) Toluidine blue staining of semi-thin cross-sections from the distal end of crush nerve. (C) Electron microscopic images of the transverse sectioned injured nerves. (D), (E) Quantification of the myelinated axon diameter and the thickness of the myelin sheaths using the Image-Pro Plus software. ${ }^{* * *} \mathrm{P}<0.001$ compared with control group, ${ }^{\# \#} \mathrm{P}<0.001$ versus PNI group, ${ }^{\boldsymbol{\Delta} \Delta} \mathbf{\Delta} \mathrm{P}<0.001$ versus bFGF group. 
bFGF-Coacervate could suppress multiple components of the ERS pathway.

\section{DISCUSSION}

Restoring the function of peripheral nerves following injury continues to be a challenging clinical problem. Currently, many protective materials and techniques are used to increase the success of nerve repair [20]. Artificial nerve conduits, typically consisting of biological materials filled with or without promoting elements, are comprehensively reported in rodent sciatic nerve injury model and human median nerve axotomy [21]. Silicone tubes, the first nerve conduits, are composed of nonresorbable polymers. They have been used to repair nerve injury for many years by increasing myelinated-axonal regeneration in an experimental 8 mm sciatic nerve gap model when filled with nerve growth factor (NGF) in the chamber $[22,23]$. However, silicone tubes have been associated with some problems including nerve compression and requiring surgery to remove the resident conduits. Polyglycolic acid (PGA) is a highly biocompatible polymer and has been approved by the U.S. Food and Drug Administration (FDA) and Conformit Europe (CE) for use in tissue engineering. Wang et al. developed an artificial nerve graft which inserted longitudinal PGA filaments to bridge dog sciatic nerve transections with a $30-\mathrm{mm}$ gap and achieved great therapeutic success [24]. However, PGA tubes are both expensive and difficult to handle during suturing, and they are only capable of bridging short nerve defects (nerve gap $<3 \mathrm{~cm}$ in length) $[25,26]$. Current research has found that collagen conduits for nerve wrapping can sustain optimal axonal growth, nerve regeneration, and functional recovery due to their low antigenicity, excellent biocompatibility, and easy accessibility. It was shown that their scaffolds can be modified with bFGF and ciliaryneurotrophic factor (CNTF) to bridge a facial nerve injury creating a $35 \mathrm{~mm}$ gap in a minipig model. The technique enhanced facial nerve reconstruction and functional recovery 6 months post-surgery [27]. However, reports highlighting the lengthy degradation period of collagen conduits showed that they could lead to chronic nerve compression at later stages [28]. Thus, while current therapies exist to treat PNI, each has drawbacks or longterm consequences that warrant investigation for new therapies to treat PNI.

Therapeutic neurotization via exogenous NTFs including bFGF, nerve growth factor (NGF), and glial cell line-derived neurotrophic factor (GDNF) have been examined extensively to cure PNI [29-31]. In contrast to other NTFs that diffuse over relatively long distances [32], bFGF exhibits a variety of biological properties including neuroprotection, neurogenesis and angiogenesis in both the central nervous system (CNS) and peripheral nervous system (PNS). Previous research has demonstrated that endogenous bFGF increased the branching of transected axonsand promoted motor functionafter facial nerve cut $[33,34]$. However, in this study, we focus on the effect of exogenous bFGF, and intrathecal administration of

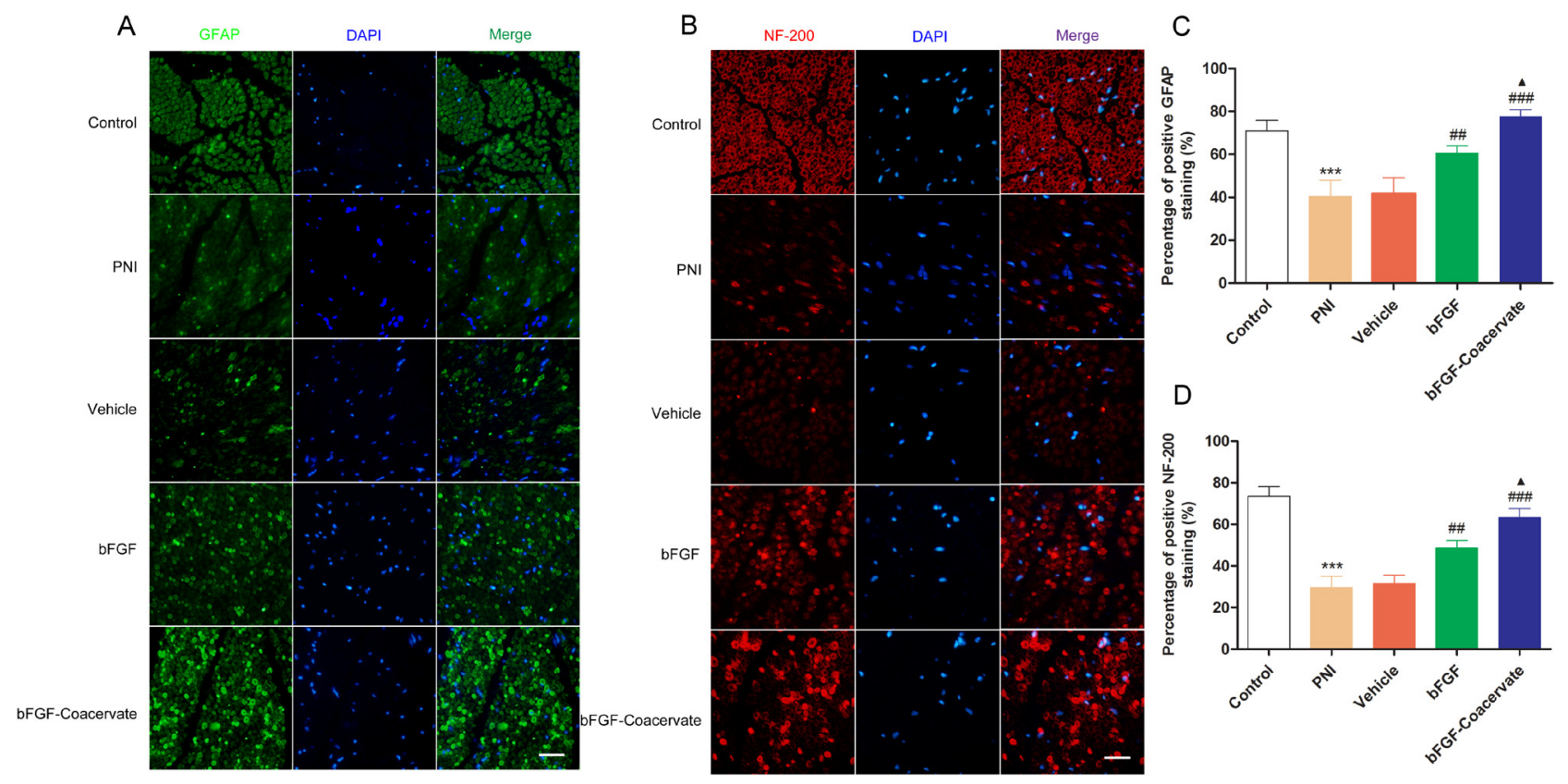

Figure 5: Evaluation of neurofilament-stained axonal growth and GFAP-positive SC proliferation in each group at 30 days post-injury. (A), (B) Immunofluorescent staining of GFAP (green), NF-200 (red), DAPI (blue) and merging of the photographs (Scale bar $=50 \mu \mathrm{m})$. (C), (D) Quantitative analysis of the GFAP and NF-200 positive staining areas in each group. ${ }^{* * *} \mathrm{P}<0.001$ compared with control group, ${ }^{\#} \mathrm{P}<0.01,{ }^{\# \#} \mathrm{P}<0.001$ versus $\mathrm{PNI}$ group, ${ }^{\mathbf{A}} \mathrm{P}<0.05$ versus bFGF group. 
bFGF could markedly alleviate functional recovery after acute spinal cord injury (SCI) [35]. Fujimoto et al. applied exogenous bFGF for situ freeze-treatment in rat saphenous nerves and observed large numbers of regenerating axons by light and electron microscopy by day 5 [36]. Several other methods utilizing bFGF were developed to overcome its short half-life and ensure adequate bFGF delivery to injured areas. For instance, Ma et al. fused linear ordered collagen scaffolds filled with native bFGF to bridge a 5-mm rat sciatic nerve gap [6], Fujimaki et al. developed a new oriented collagen tubes combined with bFGF to repair $15-\mathrm{mm}$ sciatic nerve transections in rats [37]. Both showed that the combination of bFGF with collagen enhanced axon regrowth and nerve regeneration over bFGF alone. However, whether the vehicles they used have high loading efficiency for NTFs, suitable biocompatibility, and enhanced bioactivity remains unknown.

To overcome the aforementioned challenges, we designed a new delivery vehicle-[PEAD:heparin]. In this solution system, PEAD is a polycation that can be

A Control PNI Vehicle bFGF bFGF-Coacervate
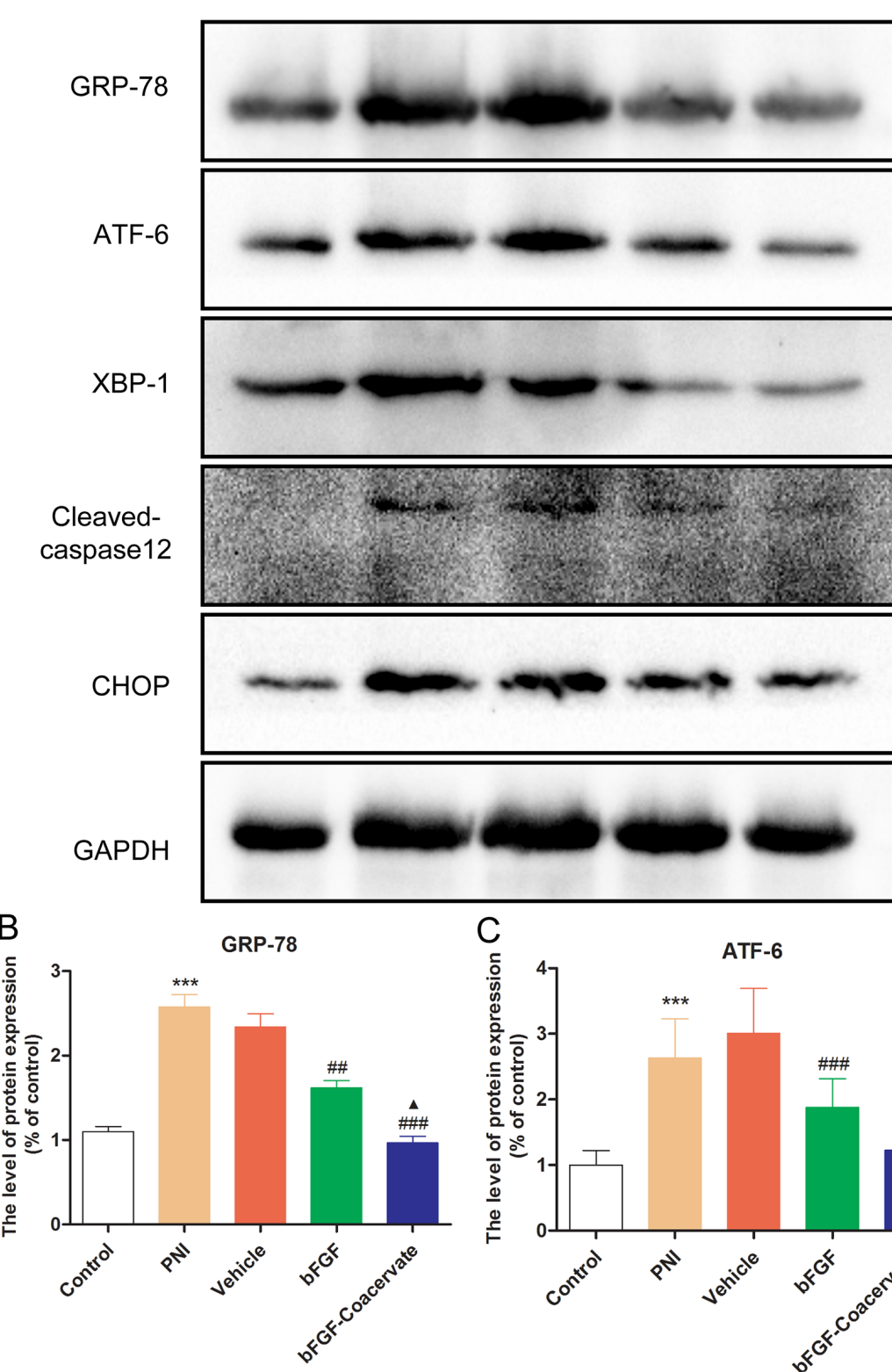

\section{$\mathrm{F}$}

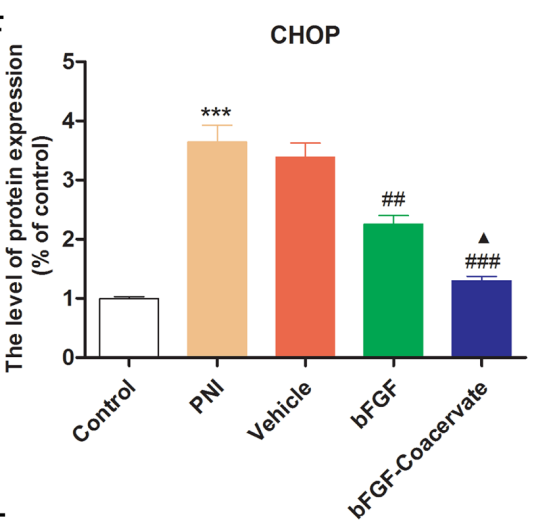

$\mathrm{E}$

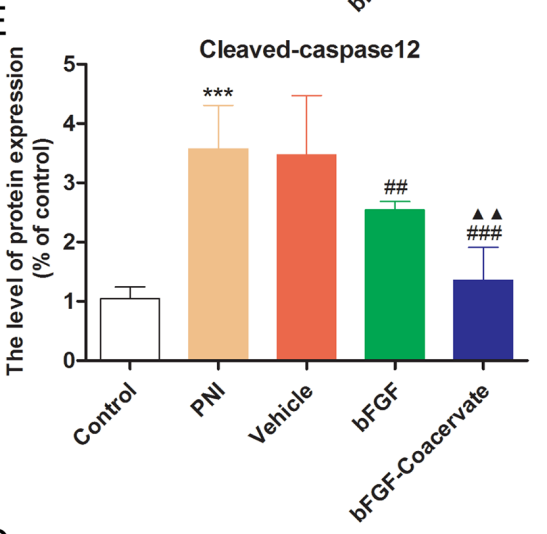

D

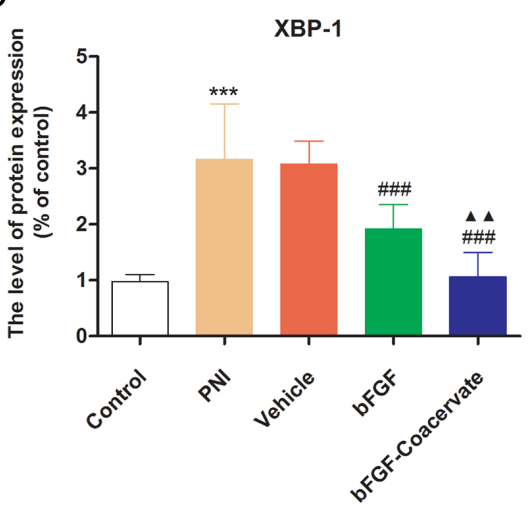

Figure 6: bFGF-coacervate administration reduced the contusion-induced ERS in injured sciatic nerve at day 30 following surgery. (A) Immunoblot for GRP-78, ATF-6, XBP-1, Cleaved-caspase 12 and CHOP in each group after treatment with [PEAD:heparin], bFGF alone, or bFGF-coacervate. GAPDH served as a protein loading control. (B-F) The optical density analysis of GRP78, ATF-6, XBP-1, Cleaved-casepase-12 and CHOP proteins. ${ }^{* * *} \mathrm{P}<0.001$ compared with control group, ${ }^{\#} \mathrm{P}<0.01$, ${ }^{\# \#} \mathrm{P}<0.001$ versus $\mathrm{PNI}$ group, ${ }^{\mathbf{\Delta}} \mathrm{P}<0.05,{ }^{\boldsymbol{\Delta}} \mathrm{P}<0.01$ versus bFGF group. 
easily synthesized, and its components including arginine, aspartic acid, glycerol, and ethylene glycol, are easily acquired. Heparin is well-studied glycosaminoglycan which can bind a variety of NTFs with high affinity. Thus, this delivery platform has the capability to conjugate bFGF by simple mixing them together. Furthermore, this ternary complex safe to use with no observable side effects, in part due to its low viscosity and good histocompatibility. In our previous study, we utilized this delivery platform to incorporate NGF, a neuroprotective factor, to repair the same crush injury model, and were able to achieve satisfactory repair as assessed by nerve regeneration and functional recovery [38]. In the present, such coacervatebFGF binding helps to not only control the release of bFGF for 35 days but also load bFGF efficiently (Figure $2 \mathrm{~B}$ and $2 \mathrm{C}$ ). With moderate sciatic nerve contusion, only one injection of the bFGF-Coacervatewas required for sustained improvement of motor and sensory function (Figure 3). Treatment with bFGF-Coacervate exhibited the strongest myelinated fiber regeneration among all injury groups (Figure 4). bFGF-Coacervate also significantly increased axonal growth and SCs proliferation in comparison to free bFGF alone (Figure 5). These results suggested that bFGF-Coacervate could enhance the neuronal restoration seen with free bFGF administration. The difference between NGF-Coacervate and bFGFCoacervate in promoting sciatic nerve recovery is due to the following two aspects. First, bFGF alone can promote nerve regeneration. However, its ability to enhance neovascularization has contributed to increased supply of nutrients and oxygen to the injury site. As a potent angiogenic factor, bFGF has a vital role in stimulating vessel sprouting and remodeling by recruiting vascular smooth muscle cells and pericytes [39, 40]. Furthermore, bFGF also triggers signaling of other angiogenic factors, which function to maintain the blood vessel networks [41], and we have demonstrated that this coacervate controlling bFGF release accelerates wound healing in full-thickness dermal wounds and maintains angiogenesis [42]. Thus, the protective effect of bFGF-Coacervate in nerve regeneration after injury is comprehensive including both neuroprotection and angiogenesis.

Accumulating evidence indicates that the functional recovery of bFGF after injury in both the PNS and CNS is related to altered levels of ERS. In our previous work, we demonstrated that the neuroprotective role of bFGF could modulated ERS in brain ischemia and reperfusion (I/R) injury and SCI $[12,43]$. Here, we also found high levels of ERS marker proteins in the injured nerve at 30 days post-surgery, leading to poor remyelination and delayed locomotor recovery. This result is contrary to that found by Maritza et al [44]. They reported that activation of ERS promoted neural regeneration and functional recovery after PNI. This discrepancy may be explained in part by their focus mainly on the endoplasmic reticulum (ER) proteostasis network in the cellular changes at the early phase. However, apoptotic pathways can be activated with excessive or prolonged ERS. Thus, we observed scarce axon regeneration and little SC proliferation 30 days post-injury, but this could be reversed with administration of bFGF-Coacervate, which suppressed ERS protein expression (Figure 6).

In summary, we report that heparin based coacervate is a promising vehicle for enhancing bFGF bioactivity by promoting neuronal recovery and remodeling after sciatic nerve crush injury. Importantly, we have implicated the inhibition of prolonged ERS as a possible mechanism. Further study is warranted to translate this technology to the clinic and combine it with multiple proteins to enhance its therapeutic effect.

\section{MATERIALS AND METHODS}

\section{Preparation of bFGF-Coacervate}

The synthesis of Poly(ethylene argininylaspartatediglyceride) (PEAD) has been reported elsewhere [45]. First, ethylene glycol diglycidyl ether and $\mathrm{t}$-BOC protected aspartic acid were polymerized through a polycondensation reaction in 1,4-dioxane to acquire the intermediate, poly(ethylene aspartate glyceride) (PED). Then, t-BOC and PED were coupled by a standard carbodiimide coupling reaction followed by the deprotection of $\mathrm{t}-\mathrm{BOC}$ to obtain PEAD. To mark the controlled delivery matrix. PEAD, heparin, and bFGF were all $10 \mathrm{mg} \mathrm{mL}^{-1}$ after dissolved in saline. Next, heparin with or without bFGF was combined at $25^{\circ} \mathrm{C}$ for $15 \mathrm{~min}$ and then mixed with PEAD to obtain [PEAD:heparin:bFGF] (bFGF-Coacervate) or [PEAD:heparin]. The ratio of PEAD to heparin was $5: 1[11]$.

\section{bFGF-Coacervate loading efficiency}

This method was conducted according to Chu et al [17]. First, heparin $100 \mu \mathrm{g}$ and 1,5 or $10 \mu \mathrm{g}$ of bFGF were mixed followed by the addition of $500 \mu \mathrm{g}$ of the PEAD solution (all solution concentrations are $10 \mathrm{mg}$ $\mathrm{mL}^{-1}$ ). Upon gently mixing, bFGF was loaded into each coacervate. Then, the ternary complex was centrifuged at $12,000 \mathrm{rpm}$ for $10 \mathrm{~min}$. Both supernatant and precipitate as well as bFGF-Coacervate without centrifugation were mixed with the corresponding dose of $5 \times$ loading buffer and boiled at $100^{\circ} \mathrm{C}$ for $10 \mathrm{~min}$. Finally, each solution was quantified by Western blotting and visualized by Quantity One $\AA$ software.

\section{bFGF release kinetics}

The release profile of bFGF from coacervate has been evaluated as described previously [42]. In brief, 0.5 $\mu \mathrm{g}$ of bFGF (10 ng $\left.\mu \mathrm{L}^{-1}\right), 10 \mu \mathrm{g}$ of heparin $\left(10 \mathrm{mg} \mathrm{mL}^{-1}\right)$, and $50 \mu \mathrm{g}$ PEAD $\left(10 \mathrm{mg} \mathrm{mL}^{-1}\right)$ were combined. After centrifugation at $12,000 \mathrm{rpm}$ for $10 \mathrm{~min}$, the supernatant 
was collected and transferred to another Eppendorf tube. $500 \mu \mathrm{L}$ saline $(0.9 \%)$ was supplied at the same time on days $1,4,7,14,21,28$, and 35 . The amount of released bFGF in the collected supernatant was measured with the bFGF ELISA kit (Westang system, Shanghai, China) and absorbance was measured at $450 \mathrm{~nm}$ with a microplate reader.

\section{PNI model and drug administration}

Male wistar rats $(200 \sim 220 \mathrm{~g})$ were obtain from the Laboratory Animals Center of Wenzhou Medical University. The living conditions and experimental procedures conformed to the National Institutes of Health (NIH) Guide Concerning the Care and Use of Laboratory Animals. All animal experiments described were approved by the Animal Experimentation Ethics Committee of Wenzhou Medical University, Wenzhou, China. All rats were kept in standardized laboratory conditions with regards to temperature $\left(23 \pm 2^{\circ} \mathrm{C}\right)$, humidity $(35-60 \%)$, and a 12:12 h light-dark cycle. Food and water were freely available to the animals for at least 7 days before the experiment.

Sciatic nerve injury was described previously [46]. Briefly, after anesthetizing with chloral hydrate (10\%, $3.5 \mathrm{~mL} \mathrm{~kg}^{-1}$ body weight) through intraperitoneal injection, the right sciatic nerves in all experimental rats were exposed by blunt dissection from the biceps femoris space. At the site $7 \mathrm{~mm}$ proximal to the bifurcation, the sciatic nerve was crushed using two vascular clips (30 g force for $2 \mathrm{~min}$, Oscar, China). The muscle layers and skin were sutured with a non-degradable suture. All rats were randomized into the following four crushed groups (PNI, vehicle, bFGF and bFGF-Coacervate) and a control group, $\mathrm{n}=8$ for each group. The bFGF-Coacervate group was administered a single intramuscular orthotopic injection of [PEAD:heparin:bFGF] coacervate solution $(300 \mu \mathrm{L})$ contained $15 \mu \mathrm{g}$ bFGF, $750 \mu \mathrm{g}$ PEAD and $150 \mu \mathrm{g}$ heparin. Similarly, the vehicle group injected the same volume of bFGF-free coacervate solution. As for the bFGF group, these rats received $500 \mathrm{ng}$ bFGF(dissolved in $100 \mu \mathrm{L}$ saline) for 30 consecutive days. Given saline as control, other groups were given the same volume of saline. After 30 days, animals were sacrificed to collect the crushed nerve and assess the pathology index.

\section{Walking track analysis}

To evaluate motor recovery in each experimental rat at $1,7,14,21$, and 28 days post-operation, walking track analysis was conducted by two independent examiners who were blinded to experiments. Individual footprints were recorded in the white paper after the rat walked through a $50 \mathrm{~cm} \times 15 \mathrm{~cm} \times 20 \mathrm{~cm}$ glass box [47] The changing parameters of paw prints were measured to calculate sciatic functional index (SFI) based on the following formula:
$\mathrm{SFI}=-38.3 \times(\mathrm{EPL}-\mathrm{NPL}) / \mathrm{NPL}+109.5 \times(\mathrm{ETS}-$ NTS $) /$ NTS $+13.3 \times($ EIT - NIT $) /$ NIT- 8.8

PL represents the distance between the third toe and heel, TS represents the distance between the first and fifth toes, IT represents the distance between the second and fourth toes, $\mathrm{E}$ is the injured right hind limb and $\mathrm{N}$ is the uninjured left hind limb. Generally, an SFI value of 0 represents normal nerve function, and SFI value around -100 indicates the sciatic nerve was crushed completely. SFI was a negative value and a higher SFI meant the better locomotive recovery of the sciatic nerve.

\section{Hot plate test}

This experiment was performed by a single investigator who was blinded to the experiment to evaluate the sensory functional recovery by measuring the rat's ability to lick and shake its hind paw on the hot plate every week following drug administration. Before testing, each group was housed in individual plastic cages to accommodate the environment for $24 \mathrm{~h}$. Then, the rats were placed on a hot plate (temperature: $55 \pm 1^{\circ} \mathrm{C}$ ). Next, the response time whenever the animals shaked or licked their paws was recorded. The cut-off time was set at 20 s to minimize skin injury. All tests were repeated 4 times with 5 min intervals. If no hind paw withdraw was observed after $20 \mathrm{~s}$, the response time was recorded as $20 \mathrm{~s}$.

\section{Histological investigation and morphometric analysis}

30 days post-surgery, animals were perfused with $4 \%$ paraformaldehyde in $0.1 \mathrm{M}$ phosphate-buffered saline (PBS), the sciatic nerve was isolated and rapidly excised at the injury sites and cut into two sections; one section (1-cm) was fixed in cold 4\% (vol/vol) paraformaldehyde overnight and then dehydrated with gradient grade ethanol, embedded in paraffin, and cut into transverse paraffin sections (5- $\mu \mathrm{m}$ thickness) for hematoxylin and eosin (H\&E) staining. The other segment (2-mm) was fixed in cold $2.5 \%$ (vol/vol) glutaraldehyde for $48 \mathrm{~h}$ and washed with PBS three times. The injured samples were immersed in $1 \%$ osmiumtetroxide solution and $1 \%$ uranyl acetate for $1 \mathrm{~h}$, respectively, dehydrated in graded acetone series, and embedded in epoxy resin. Semi-thin sections $(1-\mu \mathrm{m})$ from each segment were stained with toluidine blue (TB) and ultra-thin sections $(50-\mathrm{nm})$ of three random areas were observed under a transmission electron microscope (TEM) (H-600, HITACHI, Japan). To analyze themorphometric data of the myelin sheath thickness, the diameter was measured with Image-Pro Plus software.

\section{Immunofluorescence staining}

$5-\mu \mathrm{m}$ tissue sections in each group were dewaxed in xylene for $40 \mathrm{~min}$ and subsequently incubated in $3 \% \mathrm{H}_{2} \mathrm{O}_{2}$ and $5 \%$ bovine serum albumin (BSA) for 
30 min separately at room temperature. Sections were processed for labeling with antibodies against GFAP (1:100; Santa Cruz) and NF-200 (1:100,000; Abcam) overnight, followed by incubation with Alexa Fluor 488 or Texas Red conjugated secondary antibodies $(1: 1000$; Abcam)) for $1 \mathrm{~h}$ at room temperature. Nuclei were labeled with 4'6-Diamidino-2-phenylindole-dihydrochloride (DAPI, Beyotime Institute of Biotechnology, Shanghai, China) [48, 49]. All photographs were taken at 400× magnificationusing a Nikon ECLPSE 80i microscope (Nikon, Tokyo, Japan).

\section{Western blot analysis}

The contusion epicenter of sciatic nerve tissue $(2 \mathrm{~cm})$ was lysed in RIPA buffer containing phenylmethanesulfonyl fluoride (the volume ratio is 100:1) for $30 \mathrm{~min}$ and clarified by centrifugation at 12,000 rpm for $15 \mathrm{~min}$. Then, the extracts were quantified with Carmassi Bradford reagents (Thermo, Rockford, IL, USA). $80 \mu \mathrm{g}$ protein was separated using $12 \%$ sodium dodecyl sulfate polyacrylamide gel electrophoresis (SDS-PAGE) and electroblotted onto PVDF membranes (Millipore, USA). After blocking with 5\% skim milk for $1.5 \mathrm{~h}$, membranes were probed with primary antibody (GRP-78, ATF-6 and Cleaved-caspase 12, 1:1000, Abcam; XBP-1 and CHOP 1:300, Santa Cruz; GAPDH, 1:10000, Bioworld). Subsequently, blots were incubated for $1 \mathrm{~h}$ at room temperature with horseradish peroxidase-conjugated secondary antibodies $(1: 10000)[50,51]$. Finally, the immunoreactive bands were scanned via optical density measurements using the ChemiDoc XRS+ Imaging System (Bio-Rad). Each sample was blotted 3 independent times, and the densitometric values of the bands were quantified with the Image Lab software (Bio-Rad).

\section{Statistical analysis}

All data are shown as the mean \pm standard (SD). Statistical significance (defined as $\mathrm{P}<0.05$ ) was analyzed with GraphPad Prism 5 software (GraphPad Software Inc., La Jolla, CA, USA). Student's t-test was performed to compare the two experimental groups and one-way analysis of variance (ANOVA) with post hoc Dunnett's test was used to compare multi groups. For the walking track analysis and hot plate test, overall differences between groups were calculated using two-way ANOVA test.

\section{ACKNOWLEDGMENTS}

This study was partially supported by a research grant from the National Natural Science Funding of China (81372112), Zhejiang Provincial Program for the Cultivation of High-level Innovative Health Talents (to J.X.). the Science and technology project of Zhejiang
Province (LY17H090017 to Z.W.), J.W. would like to thank the financial support from ZhejiangProvincial Natural Science Foundation of China (LQ15E030003), Wenzhou Municipal Science \& Technology Bureau of China (Y20140574).

\section{CONFLICTS OF INTEREST}

The authors declare that they have no competing interests.

\section{REFERENCES}

1. Robinson LR. Traumatic injury to peripheral nerves. Muscle Nerve. 2000; 23: 863-73.

2. Evans GR. Challenges to nerve regeneration. Semin Surg Oncol. 2000; 19: 312-8.

3. Gordon T. The role of neurotrophic factors in nerve regeneration. Neurosurg Focus. 2009; 26: E3. doi: 10.3171/ FOC.2009.26.2.E3

4. Hernandez-Morato I, Sharma S, Pitman MJ. Changes in neurotrophic factors of adult rat laryngeal muscles during nerve regeneration. Neuroscience. 2016; 333: 44-53. doi: 10.1016/j.neuroscience.2016.07.004.

5. Wang S, Cai Q, Hou J, Bei J, Zhang T, Yang J, Wan Y. Acceleration effect of basic fibroblast growth factor on the regeneration of peripheral nerve through a 15-mm gap. J Biomed Mater Res A. 2003; 66: 522-31. doi: 10.1002/ jbm.a.10008.

6. Ma F, Xiao Z, Chen B, Hou X, Dai J, Xu R. Linear ordered collagen scaffolds loaded with collagen-binding basic fibroblast growth factor facilitate recovery of sciatic nerve injury in rats. Tissue Eng Part A. 2014; 20: 1253-62. doi: 10.1089/ten.TEA.2013.0158.

7. Ohta M, Suzuki Y, Chou H, Ishikawa N, Suzuki S, Tanihara M, Suzuki Y, Mizushima Y, Dezawa M, Ide C. Novel heparin/alginate gel combined with basic fibroblast growth factor promotes nerve regeneration in rat sciatic nerve. J Biomed Mater Res A. 2004; 71: 661-8. doi: 10.1002/ jbm.a.30194.

8. Rapraeger AC, Krufka A, Olwin BB. Requirement of heparan sulfate for bFGF-mediated fibroblast growth and myoblast differentiation. Science. 1991; 252: 1705-8.

9. Woodbury ME, Ikezu T. Fibroblast growth factor-2 signaling in neurogenesis and neurodegeneration. $\mathrm{J}$ Neuroimmune Pharmacol. 2014; 9: 92-101. doi: 10.1007/ s11481-013-9501-5.

10. Zern BJ, Chu H, Wang Y. Control growth factor release using a self-assembled [polycation:heparin] complex. PLoS One. 2010; 5: e11017. doi: 10.1371/journal.pone.0011017.

11. Chu H, Johnson NR, Mason NS, Wang Y. A [polycation:heparin] complex releases growth factors with enhanced bioactivity. J Control Release. 2011; 150: 157-63. doi: 10.1016/j.jconrel.2010.11.025. 
12. Wang Z, Zhang H, Xu X, Shi H, Yu X, Wang X, Yan Y, Fu X, Hu H, Li X, Xiao J. bFGF inhibits ER stress induced by ischemic oxidative injury via activation of the PI3K/Akt and ERK1/2 pathways. Toxicol Lett. 2012; 212: 137-46. doi: 10.1016/j.toxlet.2012.05.006.

13. Zhang H, Wu F, Kong X, Yang J, Chen H, Deng L, Cheng Y, Ye L, Zhu S, Zhang X, Wang Z, Shi H, Fu X, et al. Nerve growth factor improves functional recovery by inhibiting endoplasmic reticulum stress-induced neuronal apoptosis in rats with spinal cord injury. J Transl Med. 2014; 12: 130. doi: 10.1186/1479-5876-12-130.

14. O'Brien PD, Hinder LM, Sakowski SA, Feldman EL. ER stress in diabetic peripheral neuropathy: a new therapeutic target. Antioxid Redox Signal. 2014; 21: 621-33. doi: 10.1089/ars.2013.5807.

15. Pluquet $\mathrm{O}$, Pourtier A, Abbadie C. The unfolded protein response and cellular senescence. A review in the theme: cellular mechanisms of endoplasmic reticulum stress signaling in health and disease. Am J Physiol Cell Physiol. 2015; 308: C415-25. doi: 10.1152/ajpcell.00334.2014.

16. Zhang HY, Wang ZG, Lu XH, Kong XX, Wu FZ, Lin L, Tan X, Ye LB, Xiao J. Endoplasmic reticulum stress: relevance and therapeutics in central nervous system diseases. Mol Neurobiol. 2015; 51: 1343-52. doi: 10.1007/ s12035-014-8813-7.

17. Chu H, Gao J, Chen CW, Huard J, Wang Y. Injectable fibroblast growth factor-2 coacervate for persistent angiogenesis. Proc Natl Acad Sci U S A. 2011; 108: 134449. doi: 10.1073/pnas.1110121108.

18. Morton PD, Johnstone JT, Ramos AY, Liebl DJ, Bunge MB, Bethea JR. Nuclear factor-kappaB activation in Schwann cells regulates regeneration and remyelination. Glia. 2012; 60: 639-50. doi: 10.1002/glia.22297.

19. Hotamisligil GS. Endoplasmic reticulum stress and the inflammatory basis of metabolic disease. Cell. 2010; 140: 900-17. doi: 10.1016/j.cell.2010.02.034

20. Siemionow M, Uygur S, Ozturk C, Siemionow K. Techniques and materials for enhancement of peripheral nerve regeneration: a literature review. Microsurgery. 2013; 33: 318-28. doi: 10.1002/micr.22104.

21. Konofaos P, Ver Halen JP. Nerve repair by means of tubulization: past, present, future. J Reconstr Microsurg. 2013; 29: 149-64. doi: 10.1055/s-0032-1333316.

22. Hollowell JP, Villadiego A, Rich KM. Sciatic nerve regeneration across gaps within silicone chambers: longterm effects of NGF and consideration of axonal branching. Exp Neurol. 1990; 110: 45-51.

23. Lundborg G, Rosen B, Dahlin L, Holmberg J, Rosen I. Tubular repair of the median or ulnar nerve in the human forearm: a 5-year follow-up. J Hand Surg Br. 2004; 29: 1007. doi: 10.1016/j.jhsb.2003.09.018.

24. Wang X, Hu W, Cao Y, Yao J, Wu J, Gu X. Dog sciatic nerve regeneration across a $30-\mathrm{mm}$ defect bridged by a
chitosan/PGA artificial nerve graft. Brain. 2005; 128: 1897910. doi: 10.1093/brain/awh517.

25. Pabari A, Yang SY, Seifalian AM, Mosahebi A. Modern surgical management of peripheral nerve gap. J Plast Reconstr Aesthet Surg. 2010; 63: 1941-8. doi: 10.1016/j. bjps.2009.12.010.

26. Inada $\mathrm{Y}$, Hosoi $\mathrm{H}$, Yamashita $\mathrm{A}$, Morimoto $\mathrm{S}$, Tatsumi $\mathrm{H}$, Notazawa S, Kanemaru S, Nakamura T. Regeneration of peripheral motor nerve gaps with a polyglycolic acidcollagen tube: technical case report. Neurosurgery. 2007; 61: E1105-7; discussion E7. doi: 10.1227/01. neu.0000303210.45983.97.

27. Cui Y, Lu C, Meng D, Xiao Z, Hou X, Ding W, Kou D, Yao Y, Chen B, Zhang Z, Li J, Pan J, Dai J. Collagen scaffolds modified with CNTF and bFGF promote facial nerve regeneration in minipigs. Biomaterials. 2014; 35: 7819-27. doi: 10.1016/j.biomaterials.2014.05.065.

28. Varitimidis SE, Riano F, Vardakas DG, Sotereanos DG. Recurrent compressive neuropathy of the median nerve at the wrist: treatment with autogenous saphenous vein wrapping. J Hand Surg Br. 2000; 25: 271-5. doi: 10.1054/ jhsb.2000.0379.

29. Kemp SW, Webb AA, Dhaliwal S, Syed S, Walsh SK, Midha R. Dose and duration of nerve growth factor (NGF) administration determine the extent of behavioral recovery following peripheral nerve injury in the rat. Exp Neurol. 2011; 229: 460-70. doi: 10.1016/j.expneurol.2011.03.017.

30. Takagi T, Kimura Y, Shibata S, Saito H, Ishii K, Okano HJ, Toyama Y, Okano H, Tabata Y, Nakamura M. Sustained bFGF-release tubes for peripheral nerve regeneration: comparison with autograft. Plast Reconstr Surg. 2012; 130: 866-76. doi: 10.1097/PRS.0b013e318262f36e.

31. Tajdaran K, Gordon T, Wood MD, Shoichet MS, Borschel GH. A glial cell line-derived neurotrophic factor delivery system enhances nerve regeneration across acellular nerve allografts. Acta Biomater. 2016; 29: 62-70. doi: 10.1016/j. actbio.2015.10.001.

32. Ornitz DM, Itoh N. Fibroblast growth factors. Genome Biol. 2001; 2: REVIEWS3005.

33. Grosheva M, Nohroudi K, Schwarz A, Rink S, Bendella H, Sarikcioglu L, Klimaschewski L, Gordon T, Angelov DN. Comparison of trophic factors' expression between paralyzed and recovering muscles after facial nerve injury. A quantitative analysis in time course. Exp Neurol. 2016; 279: 137-48. doi: 10.1016/j.expneurol.2016.02.020.

34. Streppel M, Azzolin N, Dohm S, Guntinas-Lichius O, Haas C, Grothe C, Wevers A, Neiss WF, Angelov DN. Focal application of neutralizing antibodies to soluble neurotrophic factors reduces collateral axonal branching after peripheral nerve lesion. Eur J Neurosci. 2002; 15: 1327-42.

35. Rabchevsky AG, Fugaccia I, Fletcher-Turner A, Blades DA, Mattson MP, Scheff SW. Basic fibroblast growth factor (bFGF) enhances tissue sparing and functional recovery 
following moderate spinal cord injury. J Neurotrauma. 1999; 16: 817-30. doi: 10.1089/neu.1999.16.817.

36. Fujimoto E, Mizoguchi A, Hanada K, Yajima M, Ide C. Basic fibroblast growth factor promotes extension of regenerating axons of peripheral nerve. In vivo experiments using a Schwann cell basal lamina tube model. J Neurocytol. 1997; 26: 511-28.

37. Fujimaki H, Uchida K, Inoue G, Miyagi M, Nemoto N, Saku T, Isobe Y, Inage K, Matsushita O, Yagishita S, Sato J, Takano S, Sakuma Y, et al. Oriented collagen tubes combined with basic fibroblast growth factor promote peripheral nerve regeneration in a $15 \mathrm{~mm}$ sciatic nerve defect rat model. J Biomed Mater Res A. 2017; 105: 8-14. doi: 10.1002/jbm.a.35866.

38. Poggetti A, Battistini P, Parchi PD, Novelli M, Raffa S, Cecchini M, Nucci AM, Lisanti M. How to direct the neuronal growth process in peripheral nerve regeneration: future strategies for nanosurfaces scaffold and magnetic nanoparticles. Surg Technol Int. 2017; 30: pii: sti30/799.

39. Zhang J, Cao R, Zhang Y, Jia T, Cao Y, Wahlberg E. Differential roles of PDGFR-alpha and PDGFR-beta in angiogenesis and vessel stability. FASEB J. 2009; 23: 153 63. doi: 10.1096/fj.08-113860.

40. Cao R, Brakenhielm E, Pawliuk R, Wariaro D, Post MJ, Wahlberg E, Leboulch P, Cao Y. Angiogenic synergism, vascular stability and improvement of hind-limb ischemia by a combination of PDGF-BB and FGF-2. Nat Med. 2003; 9: 604-13. doi: 10.1038/nm848.

41. Cross MJ, Claesson-Welsh L. FGF and VEGF function in angiogenesis: signalling pathways, biological responses and therapeutic inhibition. Trends Pharmacol Sci. 2001; 22: 201-7.

42. Wu J, Ye J, Zhu J, Xiao Z, He C, Shi H, Wang Y, Lin C, Zhang H, Zhao Y, Fu X, Chen H, Li X, et al. Heparinbased coacervate of FGF2 improves dermal regeneration by asserting a synergistic role with cell proliferation and endogenous facilitated VEGF for cutaneous wound healing. Biomacromolecules. 2016; 17: 2168-77. doi: 10.1021/acs. biomac.6b00398.

43. Zhang HY, Zhang X, Wang ZG, Shi HX, Wu FZ, Lin BB, Xu XL, Wang XJ, Fu XB, Li ZY, Shen CJ, Li XK, Xiao J.
Exogenous basic fibroblast growth factor inhibits ER stressinduced apoptosis and improves recovery from spinal cord injury. CNS Neurosci Ther. 2013; 19: 20-9. doi: 10.1111/ cns.12013.

44. Onate M, Catenaccio A, Martinez G, Armentano D, Parsons G, Kerr B, Hetz C, Court FA. Activation of the unfolded protein response promotes axonal regeneration after peripheral nerve injury. Sci Rep. 2016; 6: 21709. doi: 10.1038/srep21709.

45. Chu H, Gao J, Wang Y. Design, synthesis, and biocompatibility of an arginine-based polyester. Biotechnol Prog. 2012; 28: 257-64. doi: 10.1002/btpr.728.

46. Li R, Wu J, Lin Z, Nangle MR, Li Y, Cai P, Liu D, Ye L, Xiao Z, He C, Ye J, Zhang H, Zhao Y, et al. Single injection of a novel nerve growth factor coacervate improves structural and functional regeneration after sciatic nerve injury in adult rats. Exp Neurol. 2017; 288: 1-10. doi: 10.1016/j.expneurol.2016.10.015.

47. Bain JR, Mackinnon SE, Hunter DA. Functional evaluation of complete sciatic, peroneal, and posterior tibial nerve lesions in the rat. Plast Reconstr Surg. 1989; 83: 129-38.

48. Feng ZC, Donnelly L, Li J, Krishnamurthy M, Riopel M, Wang R. Inhibition of Gsk3beta activity improves beta-cell function in c-KitWv/+ male mice. Lab Invest. 2012; 92: 543-55. doi: 10.1038/labinvest.2011.200.

49. Feng ZC, Li J, Turco BA, Riopel M, Yee SP, Wang R. Critical role of c-Kit in beta cell function: increased insulin secretion and protection against diabetes in a mouse model. Diabetologia. 2012; 55: 2214-25. doi: 10.1007/ s00125-012-2566-5.

50. Feng ZC, Riopel M, Li J, Donnelly L, Wang R. Downregulation of Fas activity rescues early onset of diabetes in c-Kit $(\mathrm{Wv} /+)$ mice. Am J Physiol Endocrinol Metab. 2013; 304: E557-65. doi: 10.1152/ ajpendo.00453.2012.

51. Feng ZC, Popell A, Li J, Silverstein J, Oakie A, Yee SP, Wang R. c-Kit receptor signaling regulates islet vasculature, beta-cell survival, and function in vivo. Diabetes. 2015; 64: 3852-66. doi: 10.2337/db15-0054. 